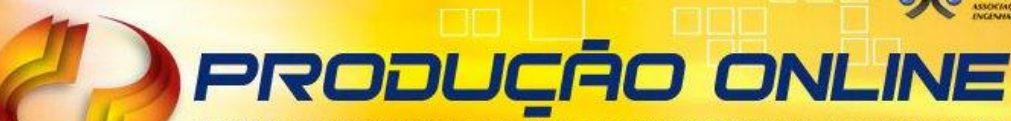 \\ REVISTA CIENTIFICA ELETRÔNICA DE ENGENHARIA DE PRODUÇÃO \\ ISSN 1676-1901
}

\section{DIMENSÕES DO APRENDIZADO ORGANIZACIONAL: VARIÁVEIS CONTEXTUAIS EM EMPRESAS EM IMPLEMENTAÇÃO ENXUTA}

\section{DIMENSIONS OF ORGANIZATIONAL LEARNING: CONTEXTUAL VARIABLES IN COMPANIES UNDER LEAN MANUFACTURING IMPLEMENTATION}

\author{
Guilherme Luz Tortorella* E-mail: gtortorella@bol.com.br \\ Flávio Sanson Fogliatto*E-mail: ffogliatto@producao.ufrgs.br \\ Giuliano Almeida Marodin* E-mail: gamarodin@gmail.com \\ João Francisco da Fontoura Vieira*E-mail: jhueao@hotmail.com \\ Juliano Denicol* E-mail: juliano denicol@hotmail.com \\ *Universidade Federal do Rio Grande do Sul (UFRGS), Porto Alegre, RS
}

\begin{abstract}
Resumo: A Produção Enxuta (PE) é uma abordagem que abrange uma variedade de práticas de gerenciamento, visando reduzir perdas e melhorar a eficiência operacional. Devido a isso, a habilidade de inovar, mudar e aprender continuamente apresenta-se como um elemento chave na implementação da PE. Diversas variáveis contextuais foram mencionadas na literatura como possíveis impedimentos para a implementação enxuta. No entanto, pouco se sabe sobre a influência dessas variáveis nas dimensões do Aprendizado Organizacional (AO). Este estudo visa examinar o relacionamento entre seis variáveis contextuais e a frequência de ocorrência de problemas em empresas que estão implementando a PE. Além disso, a identificação de relacionamentos relevantes entre dimensões do $A O$ e as variáveis contextuais contribui para a identificação de contextos nos quais se podem esperar problemas. A amostra do estudo contém treze empresas que estão implementando a PE. Os resultados indicam que as mesmas variáveis contextuais, as quais são consideradas como influentes para implementar a $\mathrm{PE}$, apresentam uma influência diferente quanto à capacidade de AO.
\end{abstract}

Palavras-Chave: Dimensões do aprendizado organizacional. Produção enxuta. Variáveis contextuais.

\begin{abstract}
The Lean Production (LP) is an approach that encompasses a variety of management practices to reduce losses and improve operational efficiency. Due to this fact, the ability to innovate, change and learn continuously presents itself as a key element in the implementation of the LP. Several contextual variables were mentioned in the literature as potential impediments to implementing lean. However, little is known about the influence of these variables on the dimensions of Organizational Learning (OL). This study aims to examine the relationship between six contextual variables and the frequency of occurrence of problems in companies that are implementing the LP. Furthermore, the identification of relevant relationships between dimensions of $\mathrm{OL}$ and contextual variables contribute to the identification of the contexts in which problems can be expected to occur. The sample contains thirteen companies implementing the LP. The results indicate that the same contextual variables, which are deemed as influential to implement LP, have a different influence on the ability of organizational learning.
\end{abstract}

Keywords: Dimensions of organizational learning. Lean production. Contextual variables.

Revista Produção Online, Florianópolis, SC, v.14, n. 3, p. 1077-1103, jul./set. 2014. 


\section{INTRODUÇÃO}

A Produção Enxuta (PE) é uma abordagem que abrange uma ampla variedade de práticas de gerenciamento, visando reduzir perdas e melhorar a eficiência operacional (SAURIN; FERREIRA, 2008). No entanto, a aplicação isolada dessas práticas não garante a implementação da filosofia enxuta. Além dos fatores técnicos, qualquer implementação da PE deve considerar os fatores intangíveis de mudanças (HUA, 2007); tais como a criação de um ambiente favorável para o desenvolvimento da aprendizagem e de lideranças na organização (BHASIN, 2011). Portanto a habilidade de inovar, mudar e aprender continuamente são elementoschave para a implementação da PE. Nesse cenário, a compreensão sobre os aspectos da Aprendizagem Organizacional (AO) e suas correlações com a PE apresenta-se como fundamental para manter as melhorias no longo prazo.

Nos últimos anos, uma considerável quantidade de artigos foi publicada focando fatores que influenciam a implementação da PE (NOGUEIRA; SAURIN, 2008; MARODIN et al., 2012; WALTER; TUBINO, 2012; FAVONI et al., 2013; MARODIN et al., 2014), os quais podem variar de um suporte gerencial (SIM; ROGERS, 2009) até as variáveis contextuais sobre as quais as empresas estão inseridas (SEZEN et al., 2011). De forma similar, o conceito de AO e seu processo têm recebido grande atenção nos campos de desenvolvimento de recursos humanos e desenvolvimento organizacional (SONG et al., 2009). Uma das questões mais críticas tem sido a falta ferramentas práticas de avaliação (KIM; MARSICK, 2013). $\mathrm{Na}$ verdade, pouco foi descoberto sobre como mensurar adequadamente a maturidade da cultura de $\mathrm{AO}$ como um processo de apoio à implementação da $\mathrm{PE}$ (TORTORELLA, 2012). Essa lacuna de pesquisa é mais significativa quando adicionadas ao problema as variáveis contextuais que influenciam as dimensões da AO em empresas sob a implementação da PE (BADRABADI; AKBARPOUR, 2013).

Esse estudo visa examinar o relacionamento entre as variáveis contextuais e a frequência de ocorrência de problemas de $A O$ em empresas que estão implementando a PE. As variáveis contextuais utilizadas foram citadas na literatura como possíveis impedimentos para a implementação da PE. Portanto, uma vez que as dimensões da $A O$ são fundamentais para o sucesso da implementação da PE (TORTORELLA, 2012), é importante entender se essas variáveis contextuais 
também influenciam a capacidade de AO das empresas. Além disso, a identificação de relacionamentos relevantes entre as dimensões de $\mathrm{AO}$ e as variáveis contextuais pode contribuir para especificar os contextos sob os quais é esperada a ocorrência de problemas.

Especificamente, o estudo foca em seis variáveis contextuais: (i) número de empregados, (ii) idade da planta, (iii) nível de sindicalização, (iv) nível educacional, (v) existência de um time melhoria contínua (MC) e (vi) receita anual. Os problemas indicados nesse estudo foram descritos de acordo com o Questionário das Dimensões de Organizações de Aprendizagem (QDOA) (MARSICK; WATKINS, 2003), o qual foi aplicado em treze empresas. Portanto algumas proposições foram investigadas com o objetivo de se aprofundar a compreensão sobre o assunto e permitir um melhor entendimento sobre as variáveis contextuais do problema. Este artigo apresenta na seção seguinte uma revisão da literatura, a qual embasa o estabelecimento das proposições. A seção 3 descreve o método empregado seguida da seção de resultados e discussão das conclusões do trabalho.

\section{REVISÃO DA LITERATURA E PROPOSIÇÕES}

\subsection{Implementação da PE e variáveis contextuais}

Em geral, o sucesso da implementação de qualquer prática de gerenciamento depende de características organizacionais e, portanto, nem todas as organizações podem ou devem implementar o mesmo conjunto de ferramentas/soluções (SHAH; WARD, 2003). Além disso, não existe uma receita única para o sucesso, uma vez que cada organização apresenta um conjunto diferente de variáveis e restrições (BHASIN, 2012b). Portanto a compreensão do contexto atual da empresa é fundamental para a adequada implementação da PE.

Variáveis contextuais representam características situacionais, as quais são, usualmente, exógenas à organização em estudo. Em muitos casos, a oportunidade de controlar ou manipular essas variáveis é, na melhor das hipóteses, limitada ou indireta; embora, em alguns casos, a organização esteja apta a realizar mudanças nessas variáveis. Este fato é apenas possível no longo prazo e através da aplicação de grande esforço (ANAND; KODALI, 2008). A Tabela 1 apresenta as variáveis 
contextuais que foram consideradas como fatores de influência para a implementação da PE e sua frequência de aparecimento na literatura.

Tabela 1 - Variáveis contextuais que influenciam a implementação da PE e sua presença na literatura

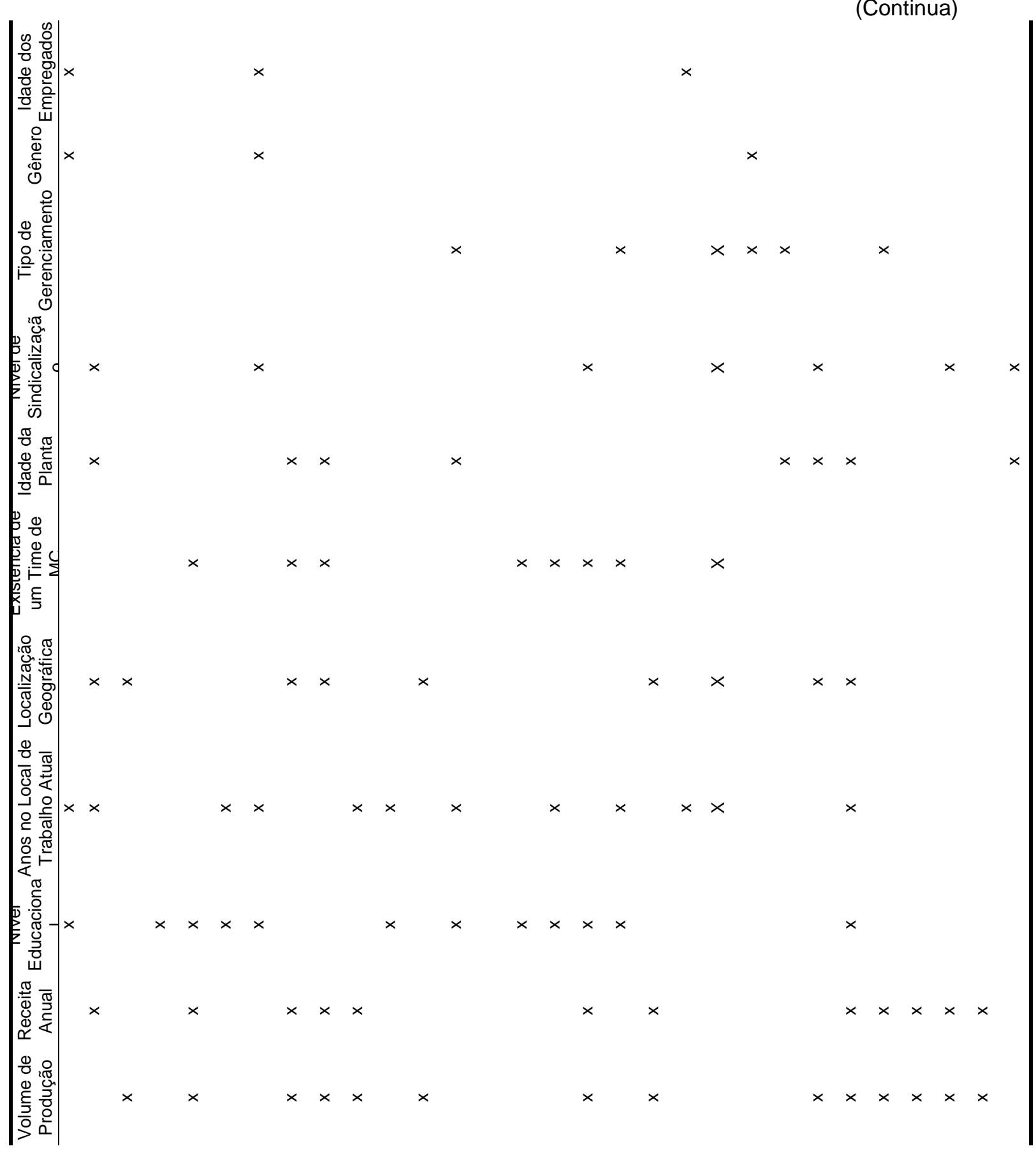


Tabela 1 - Variáveis contextuais que influenciam a implementação da PE e sua presença na literatura

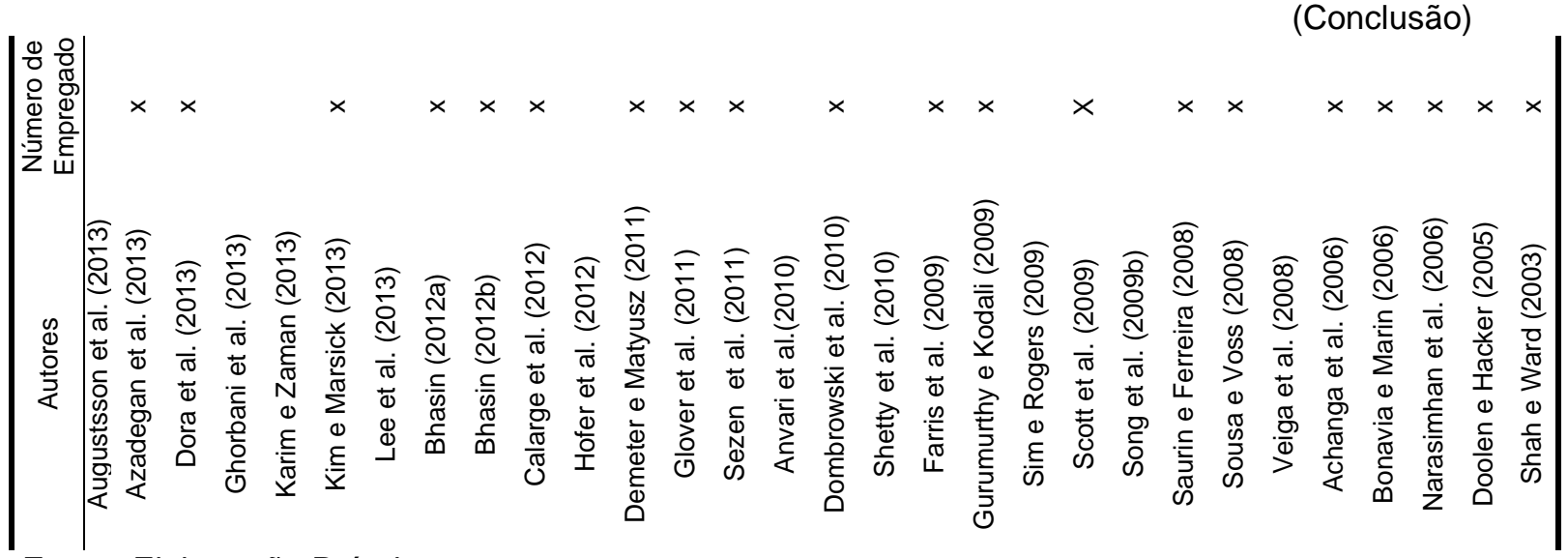

Fonte: Elaboração Própria

De acordo com a teoria de contingência, as organizações devem usar práticas da PE que são efetivas em seus contextos (ANVARI et al., 2011). Em uma implementação enxuta madura, existem quatro possíveis situações para as práticas de acordo com os seus padrões de uso e resultados de desempenho: (i) best-inclass (alto uso, alta adequação - alto desempenho), (ii) mal adequada (baixo uso, baixa adequação - baixo desempenho), (iii) panacea (alto uso, baixa adequação baixo desempenho) e (iv) promissora (baixo uso, alta adequação - alto desempenho) (SOUSA; VOSS, 2008). Portanto a abordagem de contingência assume que são as variáveis contextuais que, no longo prazo, determinam as respostas organizacionais na implementação da PE (DESAI, 2011).

\subsection{Dimensões do AO, variáveis contextuais, e proposições}

Uma vez que a implementação da $P E$ não é um evento singular, as organizações são vistas em um constante fluxo de mudança. O AO pode ser definido como "o processo de melhoria através do qual ocorre um aumento do entendimento e conhecimento dos processos organizacionais"; e está relacionado a aspectos culturais e ambientais das organizações (SONG et al., 2009). Assim, a AO pode auxiliar os funcionários a melhorarem habilidades (LEE et al., 2013) e fornecer oportunidade para encontrar melhores formas de se realizar trabalhos em grupo (SACKMANN et al., 2009).

A importância dos fatores que facilitam o $\mathrm{AO}$ tem sido ressaltada pela literatura, a qual apresenta modelos prescritivos para transformar as empresas em Revista Produção Online, Florianópolis, SC, v.14, n. 3, p. 1077-1103, jul./set. 2014. 
organizações voltadas para a aprendizagem (CHIVA et al., 2007). Consequentemente, medidas da capacidade de AO têm sido embasadas nessa literatura para determinar as dimensões ou os fatores facilitadores. Bhatnagar (2006) afirma que as capacidades de aprendizagem podem ser intangíveis ou tangíveis e podem incluir: estratégia, princípios orientadores sobre aprendizagem, estruturas, liderança, responsabilidades e funções para a aprendizagem; sistemas e processos, teorias do $\mathrm{AO}$, ferramentas, competências, recursos e valores fundamentais.

As descrições das dimensões do $\mathrm{AO}$ encontradas na literatura variam de acordo com o enfoque dado pelos pesquisadores. Hult e Ferrell (1997) classificaram suas dimensões em orientações de time, orientações de sistemas, orientação de aprendizagem e orientação de memória. O estudo de Gómez et al. (2004) identificou diferentes dimensões dentro da capacidade de $\mathrm{AO}$ : compromisso com a aprendizagem, sistemas pensantes, transferência e integração de conhecimento, abertura e experimentação. Nakhchian et al. (2013) citou que o AO pode ser propagado em grupos que tem três condições: (i) novas idéias, (ii) dúvidas sobre o conhecimento existente e as atividades da organização e (iii) desenvolver e transferir conhecimento entre o pessoal da organização.

Duas perspectivas principais surgem no seu desenvolvimento, as quais são determinadas pelos seus objetivos, que, devido ao fato de serem diferentes, possuem dimensões que diferem (LEE et al., 2013). A primeira perspectiva determina se um processo de AO está sendo realizado. Quando essa perspectiva é adotada, instrumentos para medir o AO são organizados de acordo com cada uma das fases do processo em uma tentativa de determinar sua existência dentro da organização. Essas fases são, portanto, vistas como dimensões e, futuramente, podem ser divididas em sub-componentes, tais como: experimentação, coleção de informações, análise, educação e treinamento, e transferência de informações (AUGUSTSSON et al., 2013). A segunda perspectiva visa determinar a propensão ou capacidade da organização em aprender. Quando essa perspectiva é adotada, competências que habilitam a rápida transformação organizacional são distribuídas de acordo com os principais facilitadores do $A O$, os quais são vistos como dimensões (AHMAD et al., 2013; AKSTAR et al., 2013).

Ambas perspectivas são indicações sobre comportamento individual ou social e características organizacionais; no entanto, os dois tipos de perspectivas parecem 
medir diferentes conceitos e, portanto, suas dimensões teóricas são diferentes também (GHORBANI et al., 2013). A primeira mede se o processo de AO é fluído ou está sendo completado (DESAl, 2010), enquanto que a segunda mede se a organização tem capacidade para aprender. Além disso, conclusões obtidas a partir desses dois tipos de perspectivas também diferem. Como um exemplo, Chiva et al. (2007) sugeriram que as empresas investem muito em aprendizagem individual e pouco em mecanismos para facilitar o fluxo de aprendizagem entre os níveis (individual-grupo-organizacional). Em contraste a isso, Mbengue e Sane (2013) determinaram que algumas empresas pontuaram alto ou baixo em certas características tais como "clareza dos propósitos" e "trabalho em equipe".

As dimensões utilizadas nesse trabalho são aquelas descritas no QDOA proposto por Marsick e Watkins (2003) e seguem a segunda perspectiva. No entanto, suas dimensões são obtidas a partir de uma análise abrangente de ambas as perspectivas. Além disso, nessa pesquisa, é investigado o relacionamento entre seis variáveis contextuais (retiradas das 12 apresentadas na Tabela 1), e a ocorrência de problemas em treze empresas sob a implementação da PE. Uma vez que todas as empresas estudadas estão localizadas no sul do Brasil e são independentemente gerenciadas, variáveis como "Localização geográfica" e "Tipo de gerenciamento" não foram consideradas. É importante ressaltar também que os dados coletados para as variáveis "Volume de produção, "Anos no local de trabalho atual", "Gênero" e "Idade dos empregados" não apresentaram diferenças significativas na amostra, o que fez com elas fossem descartadas da análise. Devido à natureza exploratória da pesquisa, são desenvolvidas uma série de proposições ao invés de hipóteses formais.

\subsubsection{Número de empregados}

A variável "Número de empregados" foi citada por vinte dos trinta autores examinados e está diretamente associada com o tamanho da planta (AZADEGAN et al., 2013; GLOVER et al., 2011). Seu critério de classificação pode variar de acordo com o autor (SEBRAE, 2013; CALARGE et al. 2012) embora a maioria das inferências relacionadas à variável tenham convergência e apresentem resultados coerentes (SAURIN; FERREIRA, 2008). Uma das características do AO é a fluidez 
de informações. Encorajar a disseminação efetiva de informações é decisivo para o AO porque isso pode ajudar a desenvolver um entendimento compartilhado de tarefas entre as diferentes áreas funcionais da organização (SANCHEZ et al., 2011), melhorando o conhecimento e o gerenciamento de recursos humanos da organização (NAKHCHIAN et al., 2013). Portanto existe uma crença comum entre alguns autores (DORA et al., 2013) que quanto maior o tamanho da planta, pior é o fluxo de informações, gerando efeitos negativos na capacidade de AO.

Ressalta-se, no entanto, que o grande porte de uma empresa pode implicar em disponibilidade de capital e recursos humanos, facilitando a adoção e implementação de práticas de gerenciamento de mudanças. Assim, enquanto argumentos teóricos podem ser feitos de forma a associar positiva ou negativamente empresas de grande porte e dimensões da $\mathrm{AO}$, as evidências empíricas reforçam um relacionamento positivo entre esses fatores. Ou seja, apesar da sua complexidade, grandes empresas sob a implementação da PE têm uma probabilidade menor de apresentar problemas relacionados às dimensões da AO. Então, esse trabalho apresenta a seguinte proposição, considerando o número de empregados (ou tamanho da planta):

Proposição 1: Empresas de médio porte são mais propensas a apresentar problemas de $\mathrm{AO}$ em relação às empresas de grande porte.

\subsubsection{Receita anual}

"Receita anual" é uma variável geralmente associada à quantidade de suporte financeiro que uma empresa pode fornecer para implementar a PE e seu parâmetro de classificação varia na literatura (BNDES, 2013; BHASIN, 2012a; SHETTY et al., 2010; GURUMURTHY; KODALI, 2009). Diversos pesquisadores afirmam que adotar estratégias de $\mathrm{AO}$ deveria promover o aprendizado nos níveis individual, de time e organizacional e que isso deveria produzir ganhos de desempenho financeiro (BHASIN, 2012b). Dessa forma, movimentação financeira e lucro são amplamente usados como medidas de produtividade organizacional (BHATNAGAR, 2006) e indicam a existência de certas características da visão, objetivos e estratégia de liderança de uma organização. 
Badrabadi e Akbarpour (2013) comentam que uma organização de alto desempenho formula objetivos claros e mensuráveis. Além disso, seus líderes devem orientar e garantir que o entendimento dos empregados está claro a fim de se atingir esses objetivos. Portanto, a literatura infere, empiricamente, que o processo de $A O$ é positivamente influenciado pelo desempenho financeiro da empresa (SONG et al., 2009). A partir disso, considerando a receita anual, é proposto o seguinte:

Proposição 2: Empresas com altos níveis de receita anual são menos propensas a apresentar problemas de $\mathrm{AO}$ em relação às empresas com baixos níveis de receita.

\subsubsection{Nível educacional}

Apesar da alta frequência com que aparece na literatura, "Nível Educacional" apresenta uma posição contraditória. Essa variável contextual é apresentada pelos autores como tendo tanto uma influência positiva quanto negativa no processo de aprendizado da organização. Alguns autores (AUGUSTSSON et al., 2013; GHORBANI et al., 2013) acreditam que intensificar cursos de formação por si só não é uma forma muito efetiva de mudar uma organização. Além disso, se a atitude apropriada não é reforçada, altos níveis de conhecimento podem ser uma barreira para a comunicação simples e direta dentro da organização. (KARIM; ZAMAN, 2013; FARRIS et al., 2009). No entanto a criação de programas de treinamento facilita, significativamente, a geração de redes sociais dentro da empresa, fato que é útil no desenvolvimento do $A O$, uma vez que reforça a troca de experiências entre os empregados (SANCHEZ et al., 2011).

Diversos autores mencionaram que altos níveis de educação acadêmica têm efeito positivo nos diferentes tipos de $\mathrm{AO}$, já que tal contexto habilita a introdução de novas ideias de gerenciamento, métodos e ferramentas (NAKHCHIAN et al., 2013). Além disso, um alto nível de educação pressupõe a existência de competências e conhecimentos mínimos dentro da organização, o que catalisa o processo de aprendizagem individual e, consequentemente, a capacidade de AO (BADRABADI; AKBARPOUR, 2013). A partir disso, é proposto o seguinte, considerando o nível educacional: 
Proposição 3: Empresas com alto nível educacional são menos propensas a apresentar problemas de $\mathrm{AO}$ em relação às empresas com baixo nível educacional.

\subsubsection{Existência de um time de MC}

A "Existência de um Time Formal de MC" em empresas tem sido mencionada na literatura como um facilitador para promover a aprendizagem contínua através do permanente desafio ao status quo (ROUZBAHANI et al., 2013). Além disso, alguns autores inferem que o estabelecimento de um time de $\mathrm{MC}$ com uma abordagem sistemática, que estruture as iniciativas de $\mathrm{MC}$ e favoreça o compartilhamento de conhecimento entre os indivíduos e departamentos, habilita a criação de uma massa crítica pensante dentro da organização. Este fato melhora o tipo de transferência de conhecimento do tipo prospectiva, reforçando a renovação de conhecimento dentro da organização (GARRIDO; CAMARERO, 2009). Portanto o momentum resultante impacta positivamente o $\mathrm{AO}$, uma vez que isso pode acelerar seu processo (SCOTT et al., 2009). Indícios sugerem que empresas que formalmente criam times de MC possuem vantagem natural no processo de amadurecimento das dimensões de AO. A partir disso, é proposto o seguinte, considerando a existência de um time de MC: Proposição 4: Empresas que apresentam times de MC são menos propensas a apresentar problemas de $\mathrm{AO}$ em relação às que não têm times de MC.

\subsubsection{Idade da planta}

Idade da planta pode implicar em uma tendência em direção à resistência às mudanças, uma vez que as empresas desenvolvem uma série de rotinas ou práticas organizacionais ao longo de um período de tempo e, quanto maior o tempo de experiência com as práticas, mais difícil é para mudá-las (QAHTANI; GHONEIM, 2013). A visão de "resistência às mudanças" é apresentada na literatura de sociologia organizacional que sugere que o tempo de um estabelecimento deveria influenciar inversamente na taxa de adoção de inovações (QAHTANI; GHONEIM, 2013).

Além disso, plantas antigas apresentam frequentemente uma cultura organizacional de hábitos que não favorecem a abertura, participação e Revista Produção Online, Florianópolis, SC, v.14, n. 3, p. 1077-1103, jul./set. 2014. 
transparência no time de trabalho. Portanto a idade da planta pode influenciar na flexibilidade e adaptabilidade, implicando em um impacto negativo na probabilidade de uma empresa se tornar uma organização que aprende (NARASIMHAN et al., 2006). Nesse estudo, é proposto o seguinte, considerando a idade da planta:

Proposição 5: Plantas antigas são mais propensas a apresentar problemas de AO em relação às plantas novas.

\subsubsection{Nível de sindicalização}

Supõe-se que, devido ao fato da maioria das práticas de manufatura necessitar de mudanças na organização do trabalho, empresas sindicalizadas resistirão à PE (TORTORELLA; FOGLIATTO, 2013). À medida que a implementação da PE fornece oportunidade de aprendizagem que aumenta as habilidades $e$ conhecimento do indivíduo (SCOTT et al., 2009), empregados são encorajados e capacitados a realizarem mudanças nos seus ambientes de trabalho. Porém, dessa forma, os empregados estariam agindo além do escopo tradicional de trabalho. Esse fato pode ser conflitante com os interesses do sindicato, apresentando-se, portanto, como um fator limitante para o desenvolvimento do AO (NARASIMAHAN et al., 2006).

Bhatnagar (2006) comenta que, nos anos 80, devido à pressão dos sindicatos, empresas indianas foram obrigadas a aceitar o uso altamente regulado e restritivo de investimentos domésticos e estrangeiros, o que resultou em uma inflexibilidade da força de trabalho e baixa produtividade. No entanto a sindicalização parece ser uma importante variável para apoiar algumas iniciativas de mudanças (ARMSTRONG, 2006), embora os objetivos da mudança devam estar alinhados com a política do sindicato. É importante ressaltar, no entanto, que não foi empiricamente investigada a associação desse tema com as dimensões do AO (SANCHEZ et al., 2011). A partir disso, é proposto o seguinte:

Proposição 6: Plantas sindicalizadas são mais propensas a apresentar problemas de AO em relação às não sindicalizadas. 


\section{MÉTODO}

\subsection{Variáveis contextuais}

Cada uma das variáveis contextuais são classificadas diferentemente. O tamanho da planta é classificado de acordo com SEBRAE (2013), que especifica que as empresas com mais de 500 funcionários são designadas como "grande" e plantas que possuam entre 100 e 499 funcionários como empresas de médio porte. BNDES (2013) classifica as empresas de acordo com quatro níveis de receita bruta anual: (i) "grandes" empresas apresentam receita superior a US\$100 Mi, (ii) as empresas "médias" possuem receita entre US\$30 Mi e US\$90 Mi, (iii) "pequenas" empresas apresentam receita entre US\$ $5 \mathrm{Mi}$ e US\$29 Mi e (iv) "micro" empresas têm receita menor do que US\$ 5 Mi. MTE (2013) considera uma empresa com nível de escolaridade "alto" aquela com um índice de mais de 10\% dos funcionários com diploma universitário. A existência de equipe de MC é positiva sempre que houver uma equipe formal dentro da empresa, total ou parcialmente dedicada a facilitar a implementação da PE. Se não houver evidência de uma equipe formal para facilitar a implementação da PE, então a existência da equipe de MC é negativa. Diretrizes para classificar as plantas em função da idade são escassas. IW (2013) sugere categorizar as plantas com mais de 20 anos de idade como "velhas" e plantas com menos de 10 anos de idade como "novas". Quando um sindicato representa mais de $50 \%$ da força de trabalho de produção da fábrica, a empresa classifica-se como altamente sindicalizada. Por outro lado, quando menos de $50 \%$ da força de trabalho de produção for representada por um sindicato, pode-se classificar a empresa como pouca sindicalizada.

\subsection{Características de amostra}

Os treze produtores incluídos na amostra de estudo pertencem a diferentes segmentos industriais, como mostrado na Tabela 2. Embora a implementação da PE seja geralmente associada primeiramente com fabricantes de peças discretas, a difusão de práticas em todo o espectro industrial é desconhecida (SHAH; WARD, 2007). A amostra selecionada apresenta empresas com as quais os autores Revista Produção Online, Florianópolis, SC, v.14, n. 3, p. 1077-1103, jul./set. 2014. 
estabeleceram relações de parceira acadêmica ao longo dos últimos 5 anos, possibilitando maior acesso de informações e livre trânsito dentre suas plantas e funcionários. Além disso, as empresas envolvidas têm diferentes origens culturais, já que os países onde elas começaram seus negócios são diferentes. No entanto, todos os entrevistados pertencem a plantas localizadas no Sul do Brasil e, portanto, responderam ao questionário através de uma perspectiva local, o que minimiza o efeito cultural da nacionalidade da empresa. Ocasionalmente, a amostra estudada apresenta uma quantidade equilibrada de empresas para cada variável contextual. Portanto, 54\% das empresas pesquisadas têm menos de 500 empregados e menos de $10 \%$ deles com um diploma universitário, apresentam uma receita anual inferior a US\$ $100 \mathrm{Mi}$, estabelecem uma equipe formal de MC para a implementação da PE, tem menos de vinte anos de idade e possuem um ambiente de produção sindicalizado. Todas as empresas incluídas na amostra já iniciaram a sua implementação da PE e, portanto, experimentaram algumas das barreiras e dificuldades que vão além dos fatores técnicos de produção.

Tabela 2 - Características das empresas estudadas

\begin{tabular}{ccccccccc}
\hline Comp. & $\begin{array}{c}\text { Competência } \\
\text { central }\end{array}$ & $\begin{array}{c}\text { País de } \\
\text { origem }\end{array}$ & $\begin{array}{c}\text { Número } \\
\text { de func. }\end{array}$ & $\begin{array}{c}\text { Receita } \\
\text { anual } \\
\text { (US\$) }\end{array}$ & $\begin{array}{c}\text { \% de func. } \\
\text { com diploma } \\
\text { universitário }\end{array}$ & $\begin{array}{c}\text { Existência } \\
\text { de equipe } \\
\text { de MC }\end{array}$ & $\begin{array}{c}\text { Idade da } \\
\text { planta }\end{array}$ & $\begin{array}{c}\text { Nível } \\
\text { de } \\
\text { sindic. }\end{array}$ \\
\hline A & Naval-náutica & Brasil & 451 & $29.0 \mathrm{Mi}$ & $8 \%$ & Sim & 15 anos & Baixo \\
B & Alimentícia & Brasil & 259 & $17.7 \mathrm{Mi}$ & $5 \%$ & Não & 28 anos & Alto \\
C & Alimentícia & Brasil & 459 & $22.7 \mathrm{Mi}$ & $6 \%$ & Não & 15 anos & Baixo \\
D & Automotiva & EUA & 242 & $101.4 \mathrm{Mi}$ & $16 \%$ & Não & 2 anos & Alto \\
E & Plástica & México & 487 & $20.3 \mathrm{Mi}$ & $16 \%$ & Não & 19 anos & Alto \\
F & Metalúrgica & Alemanha & 135 & $53.1 \mathrm{Mi}$ & $8 \%$ & Sim & 9 anos & Baixo \\
G & Automotiva & EUA & 423 & $48.3 \mathrm{Mi}$ & $15 \%$ & Sim & 17 anos & Alto \\
H & Automotiva & Inglaterra & 1513 & $348.7 \mathrm{Mi}$ & $18 \%$ & Sim & 40 anos & Alto \\
I & Alimentícia & EUA & 683 & $4,050 \mathrm{Mi}$ & $9 \%$ & Sim & 15 anos & Baixo \\
J & Alimentícia & Inglaterra & 915 & $708.1 \mathrm{Mi}$ & $21 \%$ & Não & 23 anos & Baixo \\
K & Metalúrgica & México & 2305 & $7,104 \mathrm{Mi}$ & $11 \%$ & Não & 101 anos & Alto \\
L & Eletrônica & Alemanha & 1604 & $152.3 \mathrm{Mi}$ & $7 \%$ & Sim & 59 anos & Alto \\
M & Metalúrgica & EUA & 519 & $90.8 \mathrm{Mi}$ & $9 \%$ & Sim & 41 anos & Alto \\
\hline
\end{tabular}

Fonte: Elaboração própria

\subsection{Instrumento e coleta de dados}

Para determinar a frequência de ocorrência de problemas $A O$, foi adaptado o QDOA originalmente desenvolvido por Marsick e Watkins (2003). O questionário 
adaptado para ser usado em um ambiente organizacional durante implantação enxuta é composto por 43 questões relacionadas às dimensões da Ao, que são divididas em três níveis de trabalho. Em cada nível, é proposto um número diferente de questões (13 para o indivíduo, 6 para a equipe, e 24 para a organização). A frequência em que ocorrem problemas de AO é avaliada usando uma escala de 6 pontos, onde 1 denota um problema que ocorre raramente e 6 denota um problema muito frequente. O questionário deve ser aplicado a indivíduos com diferentes formações e realiza-se a média das respostas obtidas a partir de cada pergunta. Em seguida, se reescala em uma escala contínua $[0,1]$ e indica-se por $\mathrm{f}_{j}(\mathrm{j}=1, \ldots, 43) \mathrm{de}$ tal modo que um valor elevado de $f_{j}$ reforça a condição desejável. Assim, a freqüência de ocorrência de problemas na empresa é dada por $h_{j}$ utilizando a seguinte expressão:

$$
h_{j}=1-f_{j}, j=1, \ldots, 43
$$

Em seguida, os valores de $h_{j}$ são agrupados de acordo com a dimensão da AO a qual a questão pertence, como mostrado na Tabela 3. O valor médio de $h_{j}$ dentro de cada grupo indica a freqüência de ocorrência de problemas em cada dimensão.

Tabela 3 - Dimensões de AO

\begin{tabular}{|c|c|c|}
\hline Dimensão & Definição & Questões \\
\hline $\begin{array}{l}1 \text { - Criar oportunidades de } \\
\text { aprendizagem contínua }\end{array}$ & $\begin{array}{l}\text { A aprendizagem é planejada de modo que os indivíduos } \\
\text { aprendam trabalhando }\end{array}$ & 1 a 7 \\
\hline 2 - Promover o diálogo & $\begin{array}{l}\text { Indivíduos obtêm habilidades para expressar suas visões e } \\
\text { capacidade para ouvir a visão alheia }\end{array}$ & 8 a 13 \\
\hline $\begin{array}{l}3 \text { - Encorajar a colaboração e a } \\
\text { aprendizagem do time }\end{array}$ & $\begin{array}{l}\text { As tarefas são concebidas para que as equipes apresentem } \\
\text { diferentes modos de pensamento; é esperado que as equipes } \\
\text { aprendam a trabalhar juntas }\end{array}$ & 14 a 19 \\
\hline $\begin{array}{l}4 \text { - Criar sistemas de captura e } \\
\text { compartilhamento da } \\
\text { aprendizagem }\end{array}$ & $\begin{array}{l}\text { Sistemas tecnológicos são criados e integrados às tarefas; } \\
\text { sistemas são mantidos e seu acesso é fornecido }\end{array}$ & 20 a 25 \\
\hline $\begin{array}{l}5 \text {-Dar autonomia aos indivíduos } \\
\text { em uma direção de visão coletiva }\end{array}$ & $\begin{array}{l}\text { O processo de decisão é descentralizado e a responsabilidade é } \\
\text { distribuída com o objetivo de estimular indivíduos a se } \\
\text { apropriarem da mudança }\end{array}$ & 26 a 31 \\
\hline $\begin{array}{l}6 \text { - Conectar a organização e seu } \\
\text { ambiente }\end{array}$ & $\begin{array}{l}\text { Indivíduos são auxiliados para perceber o efeito do seu trabalho } \\
\text { na organização como um todo }\end{array}$ & 32 a 37 \\
\hline $\begin{array}{l}7 \text { - Fornecer liderança estratégica } \\
\text { para a aprendizagem }\end{array}$ & $\begin{array}{l}\text { As lideranças utilizam a aprendizagem de uma forma } \\
\text { estratégica para gerar os resultados do negócio }\end{array}$ & 38 a 43 \\
\hline
\end{tabular}
Fonte: Adaptado de (MARSICK; WATKINS, 2003). 
Os questionários foram enviados a treze gestores de plantas diferentes, que distribuíram para os indivíduos dentro de suas organizações. O perfil de entrevistados foi selecionado aleatoriamente, incluindo diferentes níveis hierárquicos, departamentos, funções e idades. No total, cerca de 260 pessoas responderam ao questionário durante um período de coleta de dados de 3 meses. $\mathrm{A}$ unidade de análise do presente estudo é a planta de fabricação e, portanto, os dados foram organizados por empresa. A análise dos dados ocorreu através de testes de hipóteses com variância desconhecida. Esta análise permite comparação individual de cada uma das variáveis para cada dimensão de $A O$ em relação às proposições formuladas previamente na seção 2 .

\section{RESULTADOS}

\subsection{Número de empregados}

Entre as sete dimensões, uma influência significativa do tamanho da empresa só foi confirmada para a quarta dimensão, que é "Criar sistemas para capturar e compartilhar o aprendizado". Os resultados indicam que, para esta dimensão, as grandes empresas são menos propensas a apresentar problemas, como mostrado na Tabela 4. É interessante notar que essa dimensão está intimamente relacionada com os sistemas de tecnologia projetados para apoiar e facilitar o processo de aprendizagem. Normalmente, a informação e os processos de compartilhamento de conhecimento em plantas menores acontecem de uma forma mais informal do que nas empresas maiores. Este fato pode ocorrer devido a dois fatores: (i) a compreensão da empresa e a maturidade em torno da importância de um processo de compartilhamento de conhecimento estruturado (SPEAR, 2009) e (ii) a diminuição da quantidade de funcionários em si. Conseqüentemente, as plantas menores não priorizam o desenvolvimento de tal tipo de sistemas, cujo efeito é validado nos resultados do estudo. 
Tabela 4 - Resultados do teste de hipóteses para o número de funcionários (tamanho da planta)

\begin{tabular}{|c|c|c|c|c|c|c|c|}
\hline \multirow[b]{2}{*}{ Dimensão } & \multicolumn{2}{|c|}{$\begin{array}{l}\text { Companhias > } 500 \\
\text { funcionários }\end{array}$} & \multicolumn{2}{|c|}{$\begin{array}{c}\text { Companhias }<500 \\
\text { funcionários }\end{array}$} & \multirow[b]{2}{*}{$\mathrm{t}_{0}$} & \multirow[b]{2}{*}{ Teste } & \multirow[b]{2}{*}{ Status } \\
\hline & $\begin{array}{l}\text { Média da ocorr. } \\
\text { de problemas }\end{array}$ & $\begin{array}{l}\text { Desv. } \\
\text { Pad. }\end{array}$ & $\begin{array}{l}\text { Iuncion } \\
\text { Média da ocorr. } \\
\text { de problemas }\end{array}$ & $\begin{array}{l}\text { Desv. } \\
\text { Pad. }\end{array}$ & & & \\
\hline 4 & 0,31 & 0,06 & 0,41 & 0,07 & $-2,71$ & $-2,18$ & Rejeita $\mathrm{H}_{0}$ \\
\hline
\end{tabular}

Fonte: Elaboração própria.

\subsection{Receita anual}

Embora pesquisas no campo da PE demonstrarem correlação direta entre o sucesso da implementação da PE e receita anual, a variável contextual não apresenta relação significativa com as dimensões de $\mathrm{AO}$. Muitos autores propuseram uma relação positiva entre a capacidade de $\mathrm{AO}$ e vários indicadores financeiros de desempenho (receita anual, custo, retorno sobre vendas, retorno sobre o investimento, margem bruta, etc). No entanto, quando analisados separadamente, os resultados obtidos rejeitam a existência de qualquer relação com essa variável isolada. Assim, com base nesta amostra do estudo, a proposição 2 é inválida para todas as dimensões de $\mathrm{AO}$.

\subsection{Nível educacional}

Contrariamente à crença popular, os resultados mostraram que o nível educacional não tem um impacto significativo em qualquer uma das dimensões. Este resultado é um pouco surpreendente à luz da sabedoria convencional sobre a dificuldade de implementação de quaisquer práticas de gestão, sem um nível mínimo de conhecimento dentro da organização. No entanto, este resultado é consistente com os achados de Qahtani e Ghoneim (2013), que descreveram uma abordagem para transformar a Universidade Islâmica da Arábia Saudita em uma organização de aprendizagem. Apesar do alto nível de educação inerente ao contexto deste estudo de caso, os autores mencionaram que, a menos que um ambiente colaborativo e encorajador seja estabelecido, o nível educacional não vai ser um diferencial para melhorar a capacidade de AO. Além disso, eles comentam que a limitação das experiências dos indivíduos em seus departamentos é um grande obstáculo para o compartilhamento de conhecimento dentro da organização. 


\subsection{Existência de equipe de MC}

Das dimensões examinadas, a existência de equipe de MC impacta significativamente a dimensão 5, que é "Dar autonomia aos indivíduos em uma direção de visão coletiva", conforme Tabela 5. Os resultados confirmaram que as empresas que estabeleceram uma equipe formal de $M C$ são menos propensas a apresentar problemas com relação a esta dimensão do que as empresas que iniciaram a sua implementação de PE, mas não estruturaram esta equipe. Assim, é razoável esperar a presença de um processo mais robusto para o envolvimento dos indivíduos nas iniciativas de mudança.

Tabela 5 - Resultados do teste de hipóteses para existência de equipe de MC

\begin{tabular}{|c|c|c|c|c|c|c|c|}
\hline \multirow[b]{2}{*}{ Dimensão } & \multicolumn{2}{|c|}{$\begin{array}{c}\text { Companhias com equipe } \\
\text { de MC }\end{array}$} & \multicolumn{2}{|c|}{$\begin{array}{c}\text { Companhias sem equipe de } \\
\text { MC }\end{array}$} & \multirow[b]{2}{*}{$\mathrm{t}_{0}$} & \multirow[b]{2}{*}{ Teste } & \multirow[b]{2}{*}{ Status } \\
\hline & $\begin{array}{l}\text { de M } \\
\text { Média da ocorr. } \\
\text { de problemas }\end{array}$ & $\begin{array}{l}\text { Desv. } \\
\text { Pad. }\end{array}$ & $\begin{array}{l}\text { MC } \\
\text { Média da ocorr. } \\
\text { de problemas }\end{array}$ & $\begin{array}{l}\text { Desv. } \\
\text { Pad. }\end{array}$ & & & \\
\hline 5 & 0,32 & 0,03 & 0,39 & 0,05 & $-3,00$ & $-2,26$ & Rejeita $\mathrm{H}_{0}$ \\
\hline
\end{tabular}

Fonte: Elaboração própria.

\subsection{Idade da planta}

Os resultados obtidos para a variável "idade da planta" demonstraram significativa relação com a terceira dimensão chamada de "Incentivar a colaboração e o aprendizado da equipe". Não foi observada diferença estatisticamente significativa para as outras seis dimensões (ver Tabela 6). No entanto, os resultados fornecem suporte às observações sobre a dificuldade de plantas mais velhas em adaptar a sua cultura organizacional de forma a favorecer a abertura, participação e transparência do trabalho em equipe. Isto sugere que as plantas mais novas tendem a apresentar aprendizagem através da experimentação e de tentativa e erro, de forma mais ampla do que as plantas mais velhas, o que é consistente com as proposições de Wong e Tjosvold (2006). Por outro lado, surpreendentemente, a idade da planta não mostrou relação significativa com a primeira dimensão "Criar oportunidades de aprendizagem contínua", o que é contrário às considerações de Shook (2010). Este resultado indica que, embora a idade da planta tenha algum impacto sobre a capacidade de $\mathrm{AO}$, a direção do efeito não é sempre tão previsível. 
Tabela 6 - Resultados do teste de hipóteses para a idade da planta

\begin{tabular}{cccccccc}
\hline \multirow{2}{*}{ Dimensão } & \multicolumn{2}{c}{ Companhias $>20$ anos } & \multicolumn{2}{c}{ Companhias $<20$} & Anos \\
& $\begin{array}{c}\text { Média da ocorr. } \\
\text { de problemas }\end{array}$ & $\begin{array}{c}\text { Desv. } \\
\text { Pad. }\end{array}$ & $\begin{array}{l}\text { Média da ocorr. } \\
\text { de problemas }\end{array}$ & $\begin{array}{c}\text { Desv. } \\
\text { Pad. }\end{array}$ & Teste & Status \\
\hline 3 & 0,39 & 0,06 & 0,31 & 0,04 & 2,75 & 2,26 & Rejeita $\mathrm{H}_{0}$ \\
\hline
\end{tabular}

Fonte: Elaboração própria.

\subsection{Nível de sindicalização}

Em relação à variável "nível de sindicalização", os resultados apresentados na Tabela 7 indicam uma relação significativa com todos, exceto duas dimensões. Não foi encontrado um impacto significativo entre sindicalização e as dimensões "Incentivar a colaboração e o aprendizado da equipe" e "Criar sistemas para capturar e compartilhar o aprendizado". Como previsto, todas as dimensões efetuadas têm uma associação negativa significativa com o nível de sindicalização. Isso sugere que as empresas não sindicalizadas são mais propensas a apresentar capacidade de AO mais elevada do que as sindicalizadas. Além disso, esta variável contextual parece ser a mais influente na amostra do estudo e também uma variável-chave para melhorar o $A O$ nas empresas em implementação enxuta. Portanto, a proposição 6 é válida para as dimensões 1, 2, 5, 6 e 7, e é consistente com o debate na literatura (AZADEGAN et al, 2013;. LEE et al, 2013). No entanto, apesar das dificuldades esperadas que podem surgir em plantas sindicalizadas, a aprendizagem a nível da equipe não parece ser um problema, o que indica a possibilidade de adaptação dos sistemas de gestão, a fim de reforçar tais recursos e melhorar o processo de aprendizagem.

Tabela 7- Resultados do teste de hipóteses para nível de sindicalização

\begin{tabular}{cccccccc}
\hline \multirow{2}{*}{ Dimensão } & \multicolumn{2}{c}{ Companhias sindicalizas } & \multicolumn{2}{c}{ Companhias não sindicalizas } & & \multirow{2}{*}{ Teste } \\
& $\begin{array}{c}\text { Média da ocorr. } \\
\text { de problemas }\end{array}$ & $\begin{array}{c}\text { Desv. } \\
\text { Pad. }\end{array}$ & $\begin{array}{l}\text { Média da ocorr. } \\
\text { de problemas }\end{array}$ & $\begin{array}{c}\text { Desv. } \\
\text { Pad. }\end{array}$ & $\mathrm{t}_{0}$ & Teste & Status \\
\hline 1 & 0,41 & 0,06 & 0,34 & 0,05 & 2,39 & 2,18 & Rejeita $\mathrm{H}_{0}$ \\
2 & 0,38 & 0,05 & 0,30 & 0,05 & 3,20 & 2,18 & Rejeita $\mathrm{H}_{0}$ \\
5 & 0,40 & 0,05 & 0,32 & 0,07 & 2,31 & 2,23 & Rejeita $\mathrm{H}_{0}$ \\
6 & 0,45 & 0,09 & 0,32 & 0,06 & 3,00 & 2,20 & Rejeita $\mathrm{H}_{0}$ \\
7 & 0,40 & 0,07 & 0,29 & 0,07 & 2,89 & 2,18 & Rejeita $\mathrm{H}_{0}$ \\
\hline
\end{tabular}

Fonte: Elaboração própria. 


\section{CONCLUSÃO}

Esta pesquisa foi realizada utilizando os dados coletados em treze empresas localizadas no sul do Brasil. Cada uma das variáveis contextuais em estudo está associada a um conjunto de fatos significativos sobre o seu impacto na capacidade de $\mathrm{AO}$ das empresas submetidas à implementação enxuta. Abaixo, apresenta-se a discussão dos resultados e, consequentemente, as oportunidades de pesquisas futuras para o estudo.

\subsection{Discussão dos resultados}

Existem evidências na literatura que apóiam a idéia de que as grandes plantas são mais propensas a possuir os recursos para impactar positivamente as dimensões de $\mathrm{AO}$ do que plantas menores. No entanto, nossos resultados sugerem que a influência do número de empregados parece ser menos abrangente do que a sabedoria convencional infere. Na verdade, esta variável mostra apenas um impacto significativo em uma das sete dimensões. Uma explicação pode ser que, devido às características da amostra, a comparação foi feita entre empresas de grande e médio porte. Nenhuma pequena ou micro planta foi incluída na amostra do estudo. Portanto, o intervalo de comparação para o tamanho da planta foi limitado, o que pode ter influenciado os resultados obtidos para esta variável.

Os resultados sugerem que a variável receita anual, isoladamente, não influencia significativamente a ocorrência de problemas de AO dentro de uma empresa que está implementando a PE. No entanto, pesquisas anteriores mencionaram que os indicadores de desempenho financeiro, incluindo a receita anual, estão fortemente correlacionados com a capacidade de AO. Então, apesar do fato de a receita anual ser considerada como uma variável contextual única na literatura de PE, ela deve ser agrupada com os outros indicadores de desempenho financeiro, a fim de proporcionar uma comparação mais consistente. Semelhante ao caso da receita anual, a expectativa é que as empresas com maior nível educacional sejam menos propensas a apresentar problemas de $A O$ do que as empresas de nível inferior. Novamente, os resultados não suportam esta afirmação. No entanto, o conhecimento dos indivíduos é, reconhecidamente, um facilitador, especialmente 
para empresas que estão implementando a PE. Além disso, as características de certas dimensões são observadas com maior frequência em empresas com maior nível educacional.

Quanto à existência de equipe de $\mathrm{MC}$, os resultados demonstraram que esta variável influencia significativamente a freqüência de ocorrência de problemas de AO. As empresas que não possuem uma equipe de $M C$ formalmente estabelecida são mais propensas a apresentar dificuldades no que diz respeito a capacitar os indivíduos em uma direção de visão coletiva. Este fato é acentuado pela importância que esta dimensão tem para empresas que estão implementando a PE. A sustentabilidade da PE só é alcançada quando os indivíduos se comprometem com o processo de mudança e as suas iniciativas de melhoria estão alinhadas em uma direção comum.

A sabedoria convencional associa a idade da planta com uma estrutura onerosa; que as plantas mais velhas são mais propensas a apresentar problemas de $\mathrm{AO}$ do que as plantas mais novas. Na verdade, para seis das sete dimensões não houve diferença significativa na probabilidade de problemas entre as plantas mais antigas e mais recentes. No entanto, na dimensão em que as plantas mais velhas tendem a apresentar problemas, denota-se um obstáculo para o aprendizado de looping duplo, especificamente no nível da equipe, o que enfatiza a importância da adoção de práticas de gestão que o minimizem.

Finalmente, o nível de sindicalização das empresas apresentou uma associação negativa significativa com cinco dimensões de AO. Este resultado converge para as evidências anteriormente encontradas na literatura de PE. No entanto, estudos de na área de gestão afirmaram que esta variável está fortemente relacionada apenas às práticas que incluem gerenciamento de força de trabalho. Portanto, a influência negativa de sindicalização não pode ser amplamente estendida, nem para a implementação da PE nem para capacidade de $A O$.

Assim, a partir da pesquisa exploratória apresentada, pode-se compreender melhor as correlações entre as dimensões de $\mathrm{AO}$ e as variáveis contextuais organizacionais. Isto permite às empresas antever cenários sob os quais haveria possíveis dificuldades quanto à construção de uma organização de aprendizagem em uma implementação enxuta. Além disso, os resultados obtidos demonstram que os efeitos das variáveis contextuais sobre a implementação enxuta não são Revista Produção Online, Florianópolis, SC, v.14, n. 3, p. 1077-1103, jul./set. 2014. 
completamente replicáveis às dimensões de $A O$, implícitas no processo de mudança enxuta.

\subsection{Pesquisas futuras e limitações}

Embora os dados apresentem uma grande oportunidade, eles também apresentam algumas limitações. Em particular, apesar da existência de doze variáveis contextuais apontadas como influentes para a implementação da PE, só foi investigada a influência de seis delas. Portanto, a utilização de uma amostra maior permitiria uma análise mais aprofundada das outras variáveis, bem como a geração de uma perspectiva complementar do problema.

Em relação à metodologia proposta, esta investigação avaliou individualmente a influência de cada variável contextual, o que pode negligenciar a probabilidade de existência de qualquer influência interdependente entre as variáveis. Uma investigação futura pode determinar se existe qualquer efeito combinado e comparar os resultados com as relações encontradas neste estudo.

\section{REFERÊNCIAS}

ACHANGA, P.; SHEHAB, E.; ROY, R.; NELDER, G. Critical success factors for lean implementation within SMEs. Journal of Manufacturing Technology Management, v. 17, n. 4, p. 460-471. 2006. http://dx.doi.org/10.1108/17410380610662889

AHMAD, S.; MUNIAM, M.; REHMAN, A. Comparative study between two service sector organizations to measure organizational learning. International Journal of Management \& Organizational Studies, v. 2, n. 1, p. 9-21. 2013.

ANAND, G.; KODALI, R. Selection of lean manufacturing systems using the PROMETHEE. Journal of Modelling in Management, v. 3, n. 1, p. 40-70. 2008. http://dx.doi.org/10.1108/17465660810860372

ANVARI, A et al. A comparative study on journey of lean manufacturing implementation. AlJSTPME, v. 3, n. 2, p. 77-85. 2010.

ANVARI, A. et al. A proposed dynamics model for a lean roadmap. African Journal of Business Management, v. 5, n. 16, p. 6727-6737. 2011.

ARMSTRONG, M. Human Resource Management. London: Kogan Page, 2006. 
AUGUSTSSON, H.; TORNQUIST, A.; HASSON, H. Challenges in transferring individual learning to organizational learning in the residential care of older people. Journal of Health Organization and Management, v. 27, n. 3, p. 390-408. 2013. http://dx.doi.org/10.1108/JHOM-Sep-2012-0163

AZADEGAN, A. et al. The effect of environmental complexity and environmental dynamism on lean practices. Journal of Operations Management, v. 31, n.4, p. 193-212. 2013. http://dx.doi.org/10.1016/j.jom.2013.03.002

BADRABADI, H.; AKBARPOUR, T.A study on the effect of intellectual capital and organizational learning process on organizational performance. African Journal of Business Management, v. 7, n. 16, p. 1470-1485. 2013.

BHASIN, S. Measuring the Leanness of an Organization. International Journal of Lean Six Sigma, v. 2, n. 1, p. 55-74. 2011.

http://dx.doi.org/10.1108/20401461111119459

BHASIN, S. An appropriate change strategy for lean success. Management Decision, v. 50, n. 3, p. 439-458. 2012a.

http://dx.doi.org/10.1108/00251741211216223

BHASIN, S. Prominent obstacles to lean. International Journal of Productivity and Performance Management, v. 61, n. 4, p. 403-425. 2012b.

http://dx.doi.org/10.1108/17410401211212661

BHATNAGAR, J. Measuring organizational learning capability in Indian managers and establishing firm performance linkage: an empirical analysis. The Learning Organization, v. 13, n. 5, p. 416-433. 2006. http://dx.doi.org/10.1108/09696470610679965

BNDES. Banco Nacional do Desenvolvimento. 2013. Disponível em: < http://www.bndes.gov.br/SiteBNDES/bndes/bndes pt/Institucional/Apoio Financeiro/ porte.html>. Acesso em 4 Maio de 2013.

BONAVIA, T.; MARIN, J. An empirical study of lean production in the ceramic tile industry in Spain. International Journal of Operations \& Production

Management, v. 26, n. 5, p. 505-531. 2006.

http://dx.doi.org/10.1108/01443570610659883

CALARGE, F. et al. Evaluation of Lean Production System by using SAE J4000 standard: case study in Brazilian and Spanish automotive component manufacturing organizations. African Journal of Business Management, v. 6, n. 49, p. 1183911850. 2012.

CHIVA, R.; ALEGRE, J.; LAPIEDRA, R. Measuring organizational learning capability among the workforce. International Journal of Manpower, v. 28, n.3/4, p. 224-242. 2007. http://dx.doi.org/10.1108/01437720710755227

DEMETER, K.; MATYUSZ, Z. The impact of lean practices on inventory turnover. International Journal of Production Economics, v. 133, n.1, p. 154-163. 2011. http://dx.doi.org/10.1016/j.ijpe.2009.10.031 
DESAI, V. Do organizations have to change to learn? Examining the effects of technological change and learning from failures in the natural gas industry. Industrial and Corporate Change, v. 19, n. 3, p. 713-739. 2010.

http://dx.doi.org/10.1093/icc/dtp048

DESAI, V. Learning to learn from failures: the impact of operating experience on railroad accident responses. Industrial and Corporate Change, v. 20, n. 2, p. 1-28. 2011.

DOMBROWSKI, U.; CRESPO, I.; ZAHN, T. Adaptive configuration of Lean Production System in small and medium-sized enterprises. Production Engineering Research Development, v. 4, n. 4, p. 341-348. 2010.

http://dx.doi.org/10.1007/s11740-010-0250-5

DOOLEN, T.; HACKER, M. A review of lean assessment in organizations: an exploratory study of lean practices by electronics manufacturers. Journal of Manufacturing Systems, v. 24, n. 1. 2005. http://dx.doi.org/10.1016/S0278$\underline{6125(05) 80007-X}$

DORA, M. et al. Operational performance and critical success factors of lean manufacturing in European food processing SMEs. Trends in Food Science \& Technology, v. 31, n. 2, p. 1-9. 2013. http://dx.doi.org/10.1016/j.tifs.2013.03.002

FARRIS, J. et al. Critical success factors for human resource outcomes in kaizen events: an empirical study. International Journal of Production Economics, v. 117, n. 1, p. 42-65. 2009. http://dx.doi.org/10.1016/j.ijpe.2008.08.051

FAVONI, C.; GAMBI, L. N.; CARETA, C. B. Oportunidades de implementação de conceitos e ferramentas de produção enxuta visando melhoria da competitividade de empresas do APL calçadista de Jaú/SP. Revista Produção Online, v. 13, n. 3, p. 1118-1142. 2013. http://dx.doi.org/10.14488/1676-1901.v13i3.1404

GARRIDO, M.; CAMARERO, C. Assessing the impact of organizational learning and innovation on performance in cultural organizations. International Journal of Nonprofit and Voluntary Sector Marketing, v. 15, n. 3, p. 215-232. 2009.

GHORBANI, S.; DIZGAH, M.; CHEGINI, M. The relationship between intellectual capital and knowledge productivity and moderating role of organizational learning in the branches of state banks in Rasht. Journal of Basic and Applied Scientific Research, v. 3, n. 2, p. 1191-1197. 2013.

GLOVER, W. et al. Critical success factors for the sustainability of kaizen event human resource outcomes: an empirical study. International Journal of Production Economics, v. 132, n. 2, p. 197-213. 2011.

http://dx.doi.org/10.1016/j.ijpe.2011.04.005

GÓMEZ, J.; JOSÉ, J.; CÉSPEDES, L.; VALLE, C. Organizational learning capability: a proposal of measurement. Journal of Business Review, v. 58, n. 6, p. 715-725. 2004. http://dx.doi.org/10.1016/j.jbusres.2003.11.002 
GURUMURTHY, A.; KODALI, R. Benchmarking: An International Journal, v. 16, n. 2, p. 274-308. 2009.

HOFER, C.; EROGLU, C.; HOFER, A. The effect of lean production on financial performance: the mediating role of inventory leanness. International Journal of

Production Economics, v. 138, n. 2, p. 242-253. 2012.

http://dx.doi.org/10.1016/j.ijpe.2012.03.025

HUA, Y. Double-loop learning control (DLC) model for reengineering: a "yin" and "yang" balanced approach for effective organizational change. In: MANAGEMENT OF INNOVATION AND TECHNOLOGY ICMIT 2000, 1., 2000, Proceedings..., 2000. p. $116-121$.

HULT, G.; FERRELLL, O. Global organizational learning capability in purchasing: construct and measurement. Journal of Business Research, v. 40, n. 2, p. 97-111. 1997. http://dx.doi.org/10.1016/S0148-2963(96)00232-9

IW. Industry Week: manufacturing leadership excellence. 2013. Disponível em: $<$ http://www.industryweek.com/global-economy/demographics>. Acesso em 19 Julho de 2013.

KARIM, A.; ZAMAN, K. A methodology for effective implementation of lean strategies and its performance evaluation in manufacturing organizations. Business Process Management, v. 19, n. 1, p. 169-196. 2013.

http://dx.doi.org/10.1108/14637151311294912

KIM, Y.; MARSICK, V. Using the DLOQ to support learning in Republic of Korea SMEs. Advances in Developing Human Resources, v. 15, n. 2, p. 207-221. 2013. http://dx.doi.org/10.1177/1523422313475994

LEE, C. et al. Evaluating the influence of perceived organizational learning capability on user acceptance of information technology among operating room nurse staff.

Acta Anaesthesiologica Taiwanica, v. 51, n. 1, p. 22-27. 2013.

http://dx.doi.org/10.1016/j.aat.2013.03.013

MARODIN, G.; ECKERT, C. P.; SAURIN, T. A. Avançando na implantação da logística interna lean: dificuldades e resultados alcançados no caso de uma empresa montadora de veículos. Revista Produção Online, v. 12, n. 2, p. 455-479. 2012. http://dx.doi.org/10.14488/1676-1901.v12i2.932

MARODIN, G.; SAURIN, T. A.; FETTERMANN, D. C. Uma sistemática para a avaliação de riscos na implantação de produção enxuta. Revista Produção Online, v. 14, n. 1, p. 364-401. 2014. http://dx.doi.org/10.14488/1676-1901.v14.i1.1667

MARSICK, V.; WATKINS, K. Demonstrating the value of an organization's learning culture: the dimensions of the learning organization questionnaire. Advances in Developing Human Resources, v. 5, n. 2, p. 132-151. 2003.

http://dx.doi.org/10.1177/1523422303005002002 
MBENGUE, A.; SANE, S. Organizational Learning Capability: theoretical analysis and empirical study in the context of official development aid project teams.

Canadian Journal of Administrative Sciences, v. 30, n. 1, p. 26-39. 2013. http://dx.doi.org/10.1002/cjas.1238

MTE. Ministério do Trabalho e Emprego. 2013. Disponível em: <http://portal.mte.gov.br/geral/estatisticas.htm>. Acesso em 19 Julho de 2013. NAKHCHIAN, A.; ARKI, A.; BEMANI, A.The role of learning organizations in improving human resources management. European Journal of Business and Management, v. 5, n. 13, p. 159-164. 2013.

NARASIMHAN, R.; SWINK, M.; KIM, S. Disentangling leanness and agility: an empirical investigation. Journal of Operations Management, v. 24, n. 5, p. 440-457. 2006. http://dx.doi.org/10.1016/j.jom.2005.11.011

NOGUEIRA, M. G. S.; SAURIN, T. A. Proposta de avaliação do nível de implementação de típicas práticas da produção enxuta em uma empresa do setor metal-mecânico. Revista Produção Online, v. 8, n. 2, p. 1-27. 2008.

http://dx.doi.org/10.14488/1676-1901.v8i2.115

QAHTANI, F.; GHONEIM, S. Organizational learning: as an approach for transforming to learning organization concept in Saudi universities. Mediterranean Journal of Social Science, v. 4, n. 2, p. 513-532. 2013.

ROUZBAHANI, M. et al. Studying the effects of organizational learning based on organizational learning dimensions on its levels and organizational culture. Journal of Basic and Applied Scientific Research, v. 3, n. 2, p. 1297-1301. 2013.

SACKMANN, S.; REHART, P.; FRIESL, M. Sustainable Change: long-term efforts toward developing a learning organizational. Journal of Applied Behavioral Science, v. 45, n. 4, p. 521-549, 2009.

http://dx.doi.org/10.1177/0021886309346001

SANCHEZ, J.; VIJANDE, M.; GUTIERREZ, J.The effects of manufacturer's organizational learning on distributor satisfaction loyalty in industrial markets. Industrial Marketing Management, v. 40, n. 4, p. 624-635. 2011. http://dx.doi.org/10.1016/j.indmarman.2010.12.003

SAURIN, T.; FERREIRA, C. Avaliação qualitativa da implantação de práticas da produção enxuta: estudo de caso em uma fábrica de máquinas agrícolas. Gestão \& Produção, v. 15, n. 3, p. 449-462, 2008. http://dx.doi.org/10.1590/S0104$\underline{530 \times 2008000300003}$

SCOTT, B.; WILCOCK, A.; KANETKAR, V. A survey of structured continuous improvement programs in the Canadian food sector. Food Control, v. 20, n. 3, p. 209-217. 2009. http://dx.doi.org/10.1016/j.foodcont.2008.04.008

SEBRAE. Serviço Brasileiro de Apoio às Micro e Pequenas Empresas. 2013. Disponível em: http://www.sebrae.com.br/leis/. Acesso em 25 Maio de 2013. 
SEZEN, B.; KARAKADILAR, I.; BUYUKOZKAN, G. Proposition of a model for measuring adherence to lean practices: applied to Turkish automotive part suppliers. International Journal of Production Research, v. 50, n. 14, p. 1-17. 2011.

SHAH, R.; WARD, P. Defining and developing measures of lean production.Journal of Operations Management, v. 25, n. 4, p. 785-805. 2007.

http://dx.doi.org/10.1016/j.jom.2007.01.019

SHAH, R.; WARD, P. Lean manufacturing: context, practice bundles and performance. Journal of Operations Management, v. 21, n. 2, p. 129-149. 2003. http://dx.doi.org/10.1016/S0272-6963(02)00108-0

SHETTY, D.; ALI, A.; CUMMINGS, R. Survey-based spreadsheet model on lean implementation. International Journal of Lean Six Sigma, v. 1, n. 4, p. 310-334. 2010. http://dx.doi.org/10.1108/20401461011096087

SHOOK, J. How to change a culture: lessons learned from Nummi. MIT Sloan Management Review, v. 51, n. 2, p. 63-72. 2010.

SIM, K.; ROGERS, J. Implementing lean production systems: barriers to change. Management Research News, v. 32, n. 1, p. 37-49. 2009.

http://dx.doi.org/10.1108/01409170910922014

SONG, J.; JOO, B.; CHERMACK, T. The Dimensions of Learning Organization Questionnaire (DLOQ): a validation study in a Korean context. Human Resource Development Quarterly, v. 20, n. 1, p. 43-64. 2009.

http://dx.doi.org/10.1002/hrdq.20007

SOUSA, R.; VOSS, C. Contingency research in operations management practices.Journal of Operations Management, v. 26, n. 6, p. 697-713. 2008. http://dx.doi.org/10.1016/j.jom.2008.06.001

SPEAR, S. Chasing the Rabbit: how market leaders outdistance the competition and how great companies can catch up an win. New York: MacGraw-Hill, 2009.

TORTORELLA, G. Metodologia de identificação e avaliação dos fatores sócioculturais em uma empresa em implementação enxuta. $111 \mathrm{f}$. Tese (Doutorado em Engenharia de Produção) - Escola de Engenharia, Universidade Federal do Rio Grande do Sul, Porto Alegre, 2012.

TORTORELLA, G.; FOGLIATTO, F. Gestão da Mudança para um Sistema de Produção Enxuta: estado da arte e direções de pesquisa. In: SEMANA DE LA INGENIERÍA DE PRODUCCIÓN SUDAMERICANA, 13, 2013. Gramado. Anais... Gramado, 2013. 
VEIGA, G.; LIMA, E.; COSTA, S. Uma discussão sobre o papel estratégico do Modelo de Produção Enxuta. Revista Sistemas \& Gestão, v. 3, n. 2, p. 92-113. 2008.

WALTER, O. M. F. C.; TUBINO, D. F. Avaliação da implantação da manufatura enxuta: uma análise dos anais do ENEGEP. Revista Produção Online, v. 12, n. 3, p. 604-628. 2012. http://dx.doi.org/10.14488/1676-1901.v12i3.906

WONG, A.; TJOSVOLD, D. Collectivist values for learning in organizational relationships in China: the role of trust and vertical coordination. Asia Pacific Journal of Management, v. 23, n. 3, p. 299-317. 2006.

http://dx.doi.org/10.1007/s10490-006-9000-9

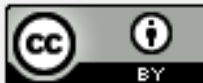

Artigo recebido em 05/09/2013 e aceito para publicação em 14/04/2014 DOI: http://dx.doi.org/ 10.14488/1676-1901.v14i3.1669 